\title{
A Comparative Histometrical Analysis of Renal Inner Medulla of Man, Swine, Dog and Hamster
}

\author{
Takeshi Kokubo, Michihito Takahashi, Fumio \\ Furukawa, Kasuke Nagano, Yuzo Hayashi and Tohru \\ TAKAHASHI* \\ Division of Pathology, National Institute for Hygienic \\ Sciences, Setagaya-ku, Tokyo 158 and * The Research \\ Institute for Tuberculosis and Cancer, Tohoku University, \\ Sendai 980
}

Kokubo, T., Takahashi, M., Furukawa, F., Nagano, K., Hayashi, Y. and Takahashi, T. A Comparative Histometrical Analysis of Renal Inner Medulla of Man, Swine, Dog and Hamster. Tohoku J. exp. Med., 1984, 142 (1), 77-88 Quantitative estimations of the total circumference (in $\mathrm{mm}$ ) of Henle's thin segment (TCH) and of the collecting tubules (TCC) in the renal inner medulla of man, swine, dog and hamster were made at different distances $(X)$ from the papillary apex. The results were expressed as exponential functions of $X$, the distance in $\mathrm{mm}$ along the medullary ray from the papillary apex to a certain point of the inner medulla, as $L=A \mathrm{e}^{B X}$. In man and swine, $\mathrm{TCH}$ was greater than TCC towards the external border of the inner medulla. However, in dog and hamster TCH was less than TCC along the entire inner medulla. The exponents of TCH showed nearly constant values (hamster : 0.68 , dog: 0.75 , man : 0.55 , swine : 0.56 ) , but those of TCC had values (hamster : 0.92, dog: 0.57, man : 0.28, swine : 0.26) correlated with the ability for concentration of urine. These findings indicate a good correlation between the ability to concentrate urine and the structure of the collecting tubules of the inner medulla, suggesting that the structure of the collecting tubules in the inner medulla affects the mechanism of urinary concentration. urinary concentration ability; total circumference of collecting tubules; exponential function

Since Hargitay and Kuhn (1951) proposed the countercurrent multiplier hypothesis in which the ascending thin segment of Henle's loop is thought to pump out sodium by active transport, it has been generally accepted that the hypertonic inner medullary interstitium results from operation of a countercurrent multiplier system in Henle's loop. On the other hand, Schmidt-Nielsen and O' Dell (1961) pointed out remarkable differences in the abilities of different mammals to concentrate urine and a close correlation of the osmolarity of the urine with the relative thickness of the medullas. However, although their 
studies indicated that the renal inner medulla participates in urinary concentration, it is still unknown, how the structure of the medullas influences the function.

The countercurrent multiplier hypothesis is based on a simplified model with a single Henle's loop. In fact, the renal medulla is a very complex structure consisting of many loops of Henle and ramified collecting tubules. Therefore, theoretical conclusions based on experimental studies on a single Henle's loop may not be applicable to medullary function. For estimation of medullary function, it seems necessary to integrate the presumed function of individual constituents of the renal inner medulla with reference to the actual structure of the medulla.

If hypertonic urine is formed as a consequence of osmotic equilibration of fluid in collecting tubules with the hypertonic medullary interstitium in the inner medulla of the kidney, the movement of water and solutes between intratubular and interstitial fluid in the inner medulla should be proportional to the area of surface of the tubular wall in contact with the fluid. Therefore, the total circumference of the collecting tubules (TCC) and of Henle's thin segments (TCH) at various points from the papillary apex are more significant than the number of tubules in estimating the total equilibration of solutes between the urinary tubules and interstitial fluid in the inner medulla.

In this study we examined the structural differences in the medullas of man, swine, dog and hamster, which have different abilities to concentrate urine. We used the relative medullary thickness (thickness of the inner medulla/thickness of the cortex), the total circumference of Henle's thin segments (TCH) and of collecting tubules (TCC) at given distances from the papillary apex as functional parameters. Data on maximal concentrating ability of man, dog and swine were obtained from the literature by Ullrich, Kramer and Boylan (1961).

\section{Materials and Methods}

Measurement of urinary osmolarity of hamsters

Ten 20-week-old hamsters ( 5 males and 5 females) were used for the mesurement of urinary osmorality. In order to obtain the concentration ability of urine, hamsters were exposed to water deprivation and salt loading. For salt loading, hamsters were maintained on commercial feed containing $100 \mathrm{~g}$ of $\mathrm{NaCl} / \mathrm{kg}$ of feed. The osmolarity of urine of each hamster was measured at 12, 24, 48, 60 and $72 \mathrm{hr}$ after water deprivation with a Advanced GigiMatic Osmometer Model 3DII manufactured by Advanced Instrument, INC (Massachusetts, USA).

\section{Kidneys}

The kidneys of a male swine (12 months old), dog (12 months old) and hamster (20 weeks old) were excised and promptly fixed in 10\% formalin solution. The kidney of a boy (1 year old) who died of acute pneumonia without apparant evidence of renal disease was also examined. The kidneys of the boy, swine, dog and hamster weighed $45 \mathrm{~g}, 100 \mathrm{~g}, 60 \mathrm{~g}$ and $0.5 \mathrm{~g}$, respectively. The tissue specimens were embedded in celloidin-paraffin.

\section{Determination of relative medullary thickness}

Frontal sections of the kidney show a distinct pattern of medullary rays from the 


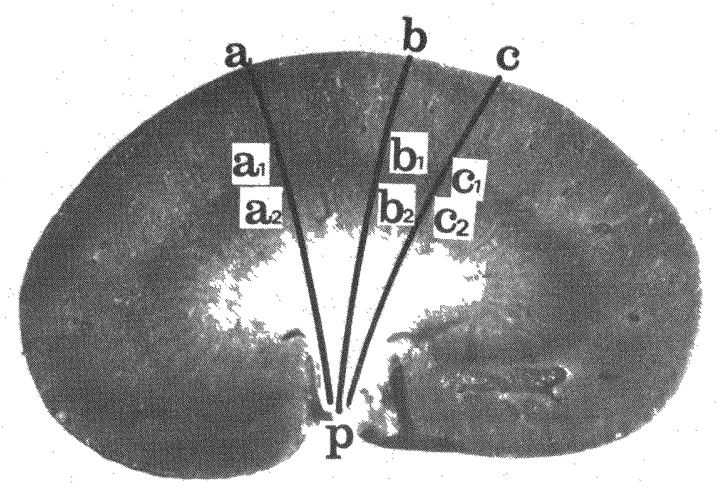

Fig. 1. Medullary rays are demonstrated on frontal section including papillary apex $(p)$ of hamster kidney. The length of the inner medulla: $\mathrm{pa}_{2}, \mathrm{pb}_{2}, \mathrm{pc}_{2}$ and, cortex : $\mathrm{aa}_{1}, \mathrm{bb}_{1}, \mathrm{cc}_{1}$.

papillary apex (Fig. 1). The cut surface was photographed and the relative medullary thickness (thickness of the inner medulla/thickness of the cortex) was measured in enlarged photographs by placing threads along the course of medullary rays. As shown in Fig. 1, medullary rays were demonstrated on frontal section including papillary apex (P). $a a_{1}, b_{1}$ and $\mathrm{cc}_{1}$ present the thickness of cortex and $\mathrm{pa}_{2}, \mathrm{pb}_{2}$ and $\mathrm{pc}_{2}$ show that of inner medulla.

\section{Preparation of serial histological sections}

The length of the medullary ray from the papillary apex to a certain point is expressed as $X$. Points with the same $X$ value on different medullary rays define a curve in the medulla, which for simplicity is hereafter called the equidistance surface. In this way the total medulla can be imagined to consist of an infinitely large number of concentric equidistance surfaces. It is expected that the proportions of different constituents are uniform and the function of urinary concentration is the same all over a certain equidistance surface. On this assumption, information on an arbitrary part of an equidistance surface

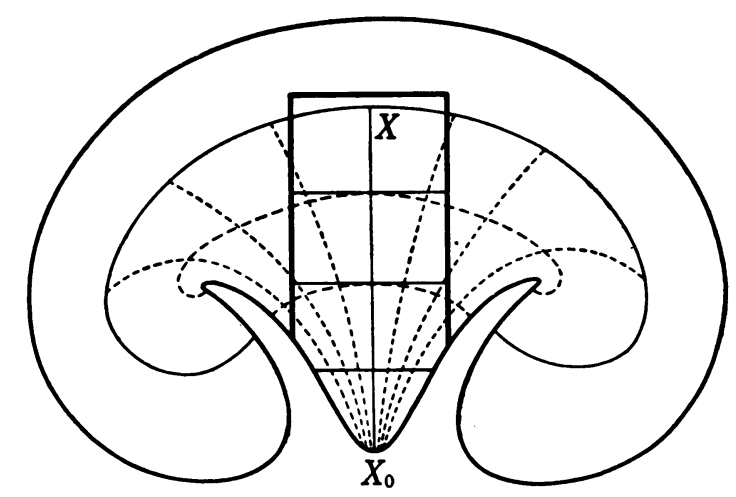

Fig. 2. The orientation of the tissue specimen for serial histological sections is illustrated with thick line. On $X_{0}-X$ the medullary ray runs straight in its entire course from medullary apex to the cortex. 
can be interpreted as a representative of the whole equidistance surface. As shown in Fig. 2 , in the middle of the corn of the kidney, the medullary rays delineate a straight line from the medullary apex towards the corticomedullary border. Serial histological sections of this part demonstrate exact transverse tubular sections of the entire course of tubules in the medulla. So with all kidneys, serial sections $3 \mu \mathrm{m}$ in thickness of this part were cut perpendicular from the medullary apex towards the cortex. Although the curved equidistance surface is not strictly equal to the plane of histological sections, the divergence of collecting tubules in the part of the medulla examined was limited to the range of negligible. The sections were stained with a mixture of Bieblich's scarlet and aniline blue.

Determination of the circumferences of individual collecting tubules (CIC) and individual Henle's thin segments (CIH)

The basement membrane of the collecting tubules and Henle's thin segments was stained as a sharp line, but the plasma membrane on the luminal side could not be seen as a line with any histological staining method used. Therefore, for technical reasons, the external border was used to measure the circumference of the collecting tubules and Henle's thin segments.

The basement membranes in exact transverse sections of collecting tubules and Henle's thin segments at different $X$ levels were depicted on tracing paper at $\times 500$ magnification with a Nikon projector, Model V-16 (Nikon, Tokyo) and then the circumference of the collecting tubule and the circumference of the Henle's thin segment were measured with a Digigram, Model-GS (Muto Ind. Co., Ltd., Tokyo). Average values of circumferences of 50 to 100 collecting tubules and Henle's thin segments, respectively, were calculated.

Determination of the total number and the total circumference of the collecting tubules (TCC)

The total number of the collecting tubules was calculated as follow:

The inner medulla was divided into a large number of equidistance surfaces, each separated by an equal distance $\boldsymbol{\Delta} X$. The equidistance surfaces were numbered from the medullary apex to the border of the inner and outer medulla. In the range of one quarter of the thickness of the inner medulla from the papillary apex, the total number of the collecting tubules $N_{i}$ could be determined directly on the $i$-th equidistance surface. On this surface a certain number of collecting tubules, $m_{i}$, was taken at random. By graphical reconstruction from the serial histological sections, the selected number of collecting tubules were pursued for the number, $m_{i+1}$, generated from them after a course $\boldsymbol{\Delta} X$. The ratio $m_{i+1} /$ $m_{i}=p_{i}$. If the total number of the collecting tubules on the $i$-th surface is $N_{i}$ that on the $(i+j)$-th equidistance surface is given by

$$
N_{i+j}=p_{1} \cdot p_{2} \cdot p_{3} \cdots \cdots N_{i}
$$

Consequently, the total number $N_{t}$ of collecting tubules could be defined by a function of $X$. In this study, the total circumferences of the collecting tubules (TCC) and of the thin segments of Henle's loop (TCH) are used as parameters of the physiological function of the inner medulla. TCC at a certain $X$ could be obtained as the product of the circumference of an individual collecting tubule (CIC) and the total number $N_{t}$ of collecting tubules.

Determination of the total number and the total circumference of thin segments of Henle's loop (TCH)

The total number of Henle's thin segments $N_{h}$ was determined as follow : An eye-piece with a lattice was superposed on the microscopical field, and the numbers of Henle's thin segments and collecting tubules in a given surface area were counted. The ratio $N_{i} / N_{h}=$ $K_{i}$. $N_{h}$ could be obtained when $N_{i}$ was known. From the product of $N_{h}$ and an individual circumference of Henle's thin segment $(\mathrm{CIH})$, the total circumference of Henle's thin 
segments at a certain $X$ could be determined. The total circumference of the ascending limbs or descending limbs of Henle's loop were given by a half the product of $N_{h}$ and CIH. In this study $\mathrm{TCH}$ means $1 / 2 \cdot N_{h} \bullet \mathrm{CIH}$.

\section{Results}

Urinary osmolarity of hamsters

Urinary osmolarity of salt loading hamsters at 12, 24, 48, 60 and $72 \mathrm{hr}$ after water deprivation are shown in Table 1

TABLE 1. Urine osmolarity $\left(\mathrm{mOsm} / \mathrm{kg} . \mathrm{H}_{2} \mathrm{O}\right)$ of hasters at different times after water deprivation

\begin{tabular}{crrrrr}
\hline $\begin{array}{c}\text { Animal } \\
\text { No. }\end{array}$ & $12 \mathrm{hr}$ & $24 \mathrm{hr}$ & $48 \mathrm{hr}$ & $60 \mathrm{hr}$ & $72 \mathrm{hr}$ \\
\hline 1 & 1074 & 1178 & 1972 & 1816 & 2062 \\
2 & 1082 & 1246 & 2314 & 2428 & 2314 \\
3 & 918 & 1468 & 1966 & 2314 & 2318 \\
4 & 868 & 1032 & 1848 & 1966 & 2458 \\
5 & 649 & 756 & 1822 & 1948 & 2369 \\
6 & 1466 & 1471 & 1918 & 1882 & 2212 \\
7 & 474 & 690 & 1414 & 1918 & 1962 \\
8 & 842 & 938 & 2102 & 2216 & 2310 \\
9 & 1042 & 1148 & 1842 & 2402 & 2602 \\
10 & 824 & 1422 & 1802 & 2349 & 2421 \\
\hline Mean \pm s.D. & $924 \pm 270$ & $1135 \pm 281$ & $1900 \pm 231$ & $2123 \pm 240$ & $2303 \pm 187$ \\
\hline & * Standard deviation & & &
\end{tabular}

* Standard deviation.

\section{Relative medullary thickness}

In Fig. $2, \frac{a_{2} p}{a_{1}}: \frac{b_{2} p}{b b_{1}}: \frac{c_{2} p}{c c_{1}}$ was almost constant in all subjects examined. (man : $1.40: 1.30: 1.44$, swine : $0.51: 0.49: 0.47$, dog: $1.39: 1.50: 1.55$, hamster : $2.69: 2.74: 2.67)$. It is concluded that the relative medullary thickness was essentially the same in all directions. Values for the relative medullary thickness

TABLE 2. The relative medullary thickness (length of the inner zone/length of the cortex)

\begin{tabular}{lcccc}
\hline Species & Weight $(\mathrm{g})$ & Cortex $(\mathrm{mm})$ & $\begin{array}{c}\text { Inner } \\
\text { zone }(\mathrm{mm})\end{array}$ & $\begin{array}{c}\text { Inner } \\
\text { zone/Cortex }\end{array}$ \\
\hline Man & 45.0 & 4.0 & 5.5 & 1.38 \\
Swine & 120.0 & 6.7 & 3.3 & 0.49 \\
Dog & 60.0 & 2.5 & 3.7 & 1.48 \\
Hamster & 0.5 & 1.0 & 2.7 & 2.27 \\
\hline
\end{tabular}


of the inner medulla (i.e., the ratio of the thickness of the inner medulla and cortex) for the kidneys examined are shown in Table 2 . The values increas in the order, hamster, dog, man and swine.

\section{Total numbers of Henle's thin segments (TNH) and collecting tubules (TNC)}

The total number of collecting tubules (TNC) and the total number of Henle's thin segments (TNH) are shown in Fig. 3. With a semilogarithmic coordinate system, a distinct linear regression was observed between the values of the animals or human case examined. As shown in Fig. 3, TNC was more than TNH in the dog and hamster and $\mathrm{TNC}$ was less than $\mathrm{TNH}$ in man and swine along the entire length of the inner medulla. A marked deflection of the regression line was found at about one quarter of the height of the inner medulla in all species, but its significance is unknown.
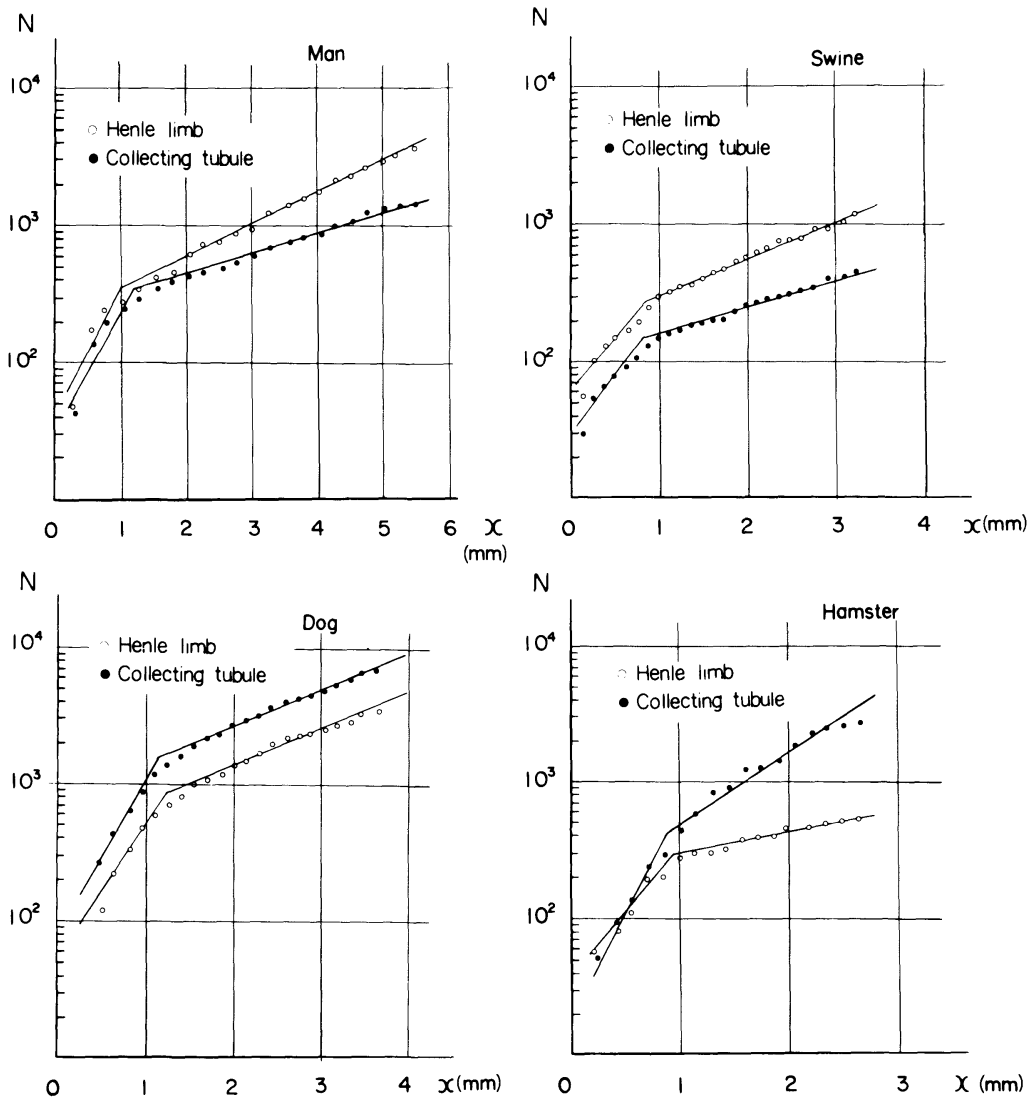

Fig. 3. The total number $\mathrm{N}$ of the collecting tubules (TNC) and of Henle's thin segments (TNH) on a semilogarithmic coordinate system are shown. TNH more than TNC in man and swine and TNH less than TNC in dog and hamster along entire inner medulla are demonstrated. Note a deflection of regression line at a quater length from papillary apex in all animals. 
Circumference of individual collecting tubule (CIC)

Results on the individual circumference of collecting tubules are shown in Fig. 4. A distinct negative linear regression was demonstrated on a semilogarithmic coordinate system, and the equations obtained, expressed as exponential functions of $X$, are given in Table 3 .
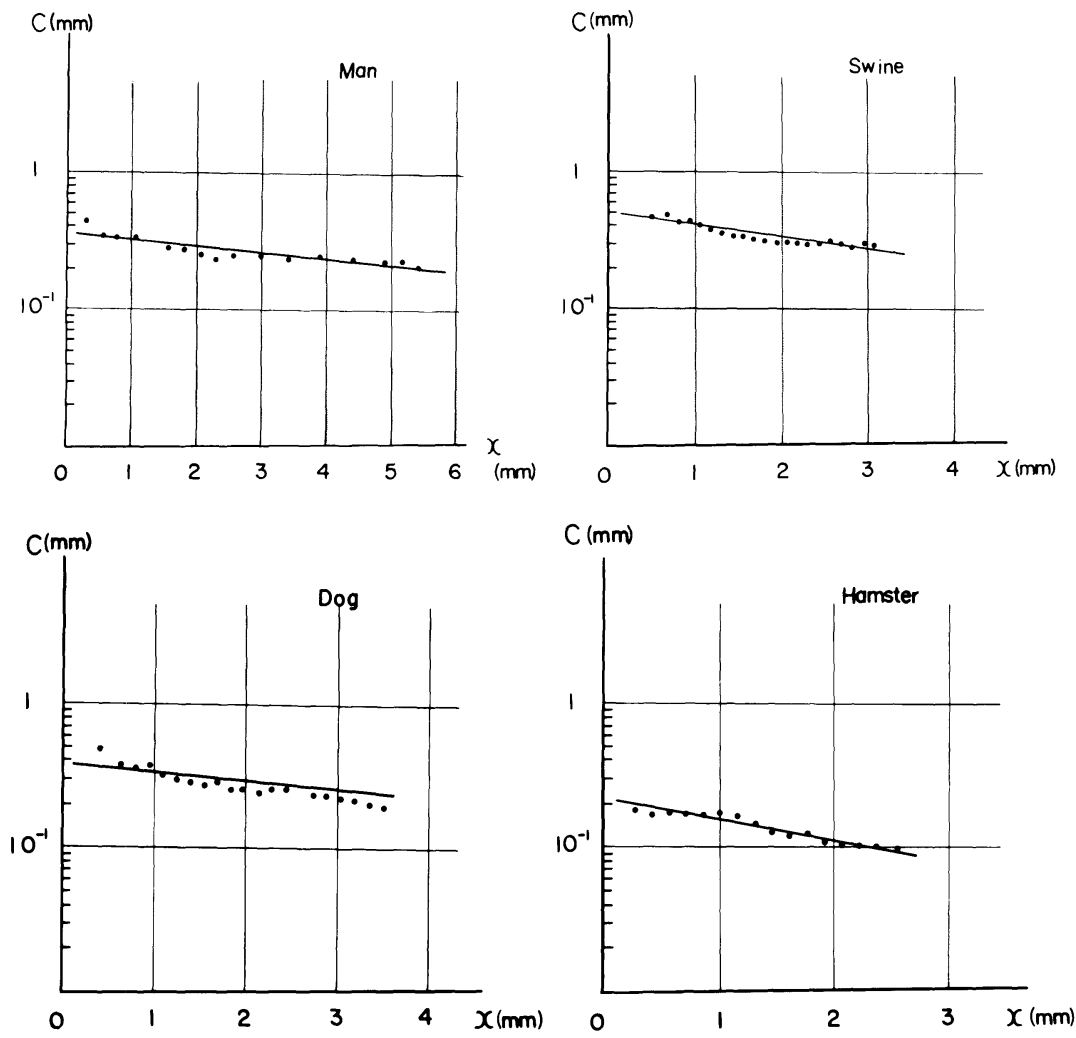

Fig. 4. External circumference $\mathrm{C}$ of individual collecting tubules is presented against $X$ on a semilogarithmic coordinate system. Note distinct linear regression. Each dot represents the mean of measurements on about 50 to 100 traverse sections of the collecting tubules from the same histological section.

TABLE 3. The equations of external circumference $C$ of the individual collecting tubule expressed as a function of $X$

\begin{tabular}{lll}
\hline \multicolumn{1}{c}{ Species } & $C=A \mathrm{e}^{B X}(\mathrm{~mm})$ & $Y=B X+K(Y-\log C)$ \\
\hline Man & $C=0.35 \mathrm{e}^{-0.11 X}$ & $Y=-0.11 X-1.05$ \\
Swine & $C=0.50 \mathrm{e}^{-0.21 X}$ & $Y=-0.21 X-0.69$ \\
Dog & $C=0.37 \mathrm{e}^{-0.06 X}$ & $Y=-0.06 X-0.99$ \\
Hamster & $C=0.31 \mathrm{e}^{-0.30 X}$ & $Y=-0.30 X-1.57$
\end{tabular}

$A, B$ and $K$ are constants. 
TABLE 4. The equations of total external circumference $L$ of the collecting tubules (TCC) expressed as a exponential function of $X$

\begin{tabular}{lll}
\hline Species & $L=A \mathrm{e}^{B X}(\mathrm{~mm})$ & $Y=B X+C(Y=\log L)$ \\
\hline Man & $L=64.85 \mathrm{e}^{0.28 X}$ & $Y=0.28 X+4.17$ \\
Swine & $L=45.79 \mathrm{e}^{0.26 X}$ & $Y=0.26 X+3.82$ \\
Dog & $L=190.78 \mathrm{e}^{0.57 X}$ & $Y=0.57 X+5.25$ \\
Hamster & $L=27.57 \mathrm{e}^{0.92 X}$ & $Y=0.92 X+3.32$ \\
\hline
\end{tabular}

$A, B$ and $C$ are constants.
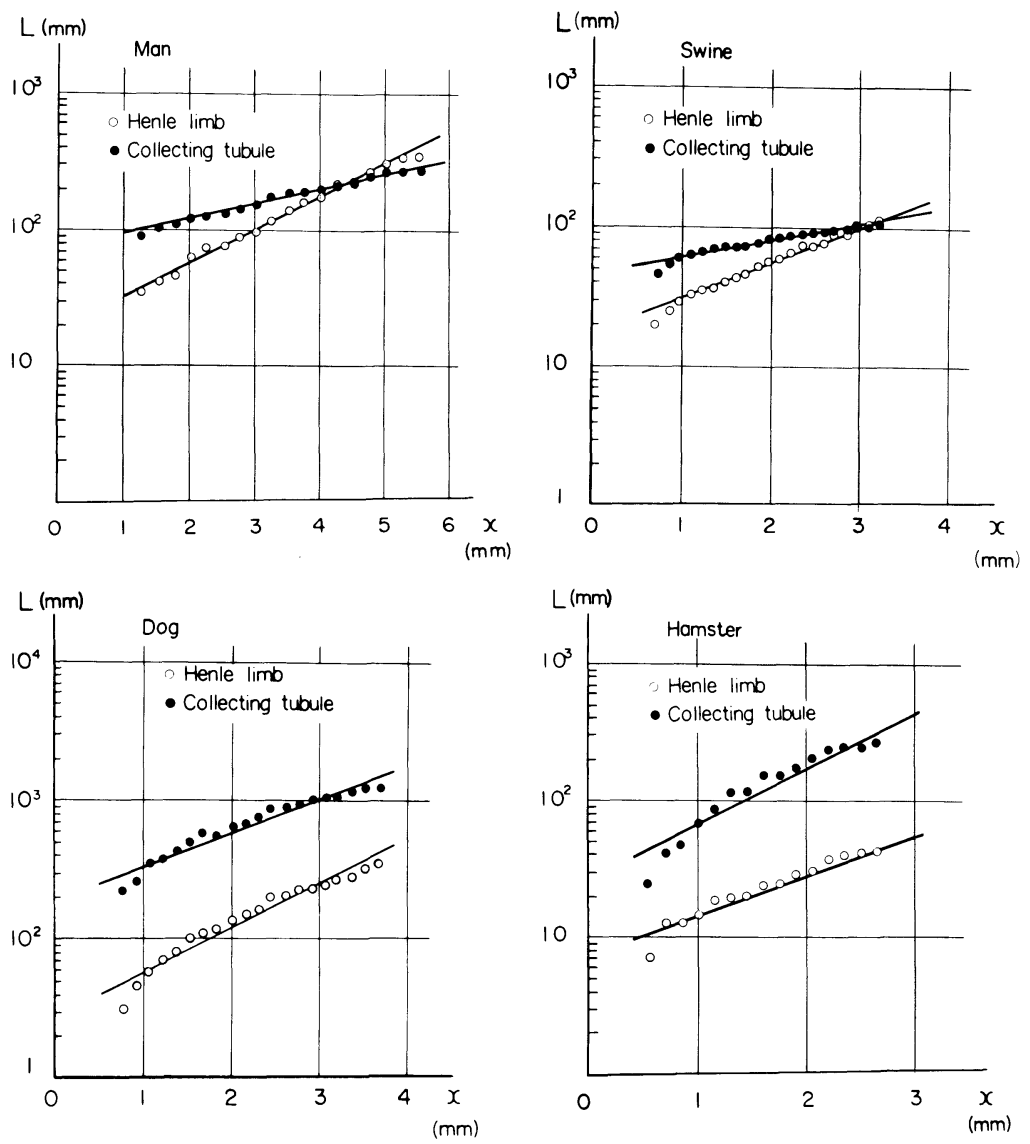

Fig. 5. Total external circumference L of the collecting tubules (TCC) and of Henle's thin segments $(\mathrm{TCH})$ are presented against $X$ on a semilogrithmic coordinate system. TCH greater than TCC toward corticomedullary border is demonstrated in man and swine. In contrast, TCH less than TCC along entire inner medulla is shown in dog and hamster. 
Total circumference of collecting tubules (TCC)

A distinct linear regression was obtained on a semilogarithmic coordinate system for the total circumference of collecting tubules in the animals or human case examined (Fig. 5). As shown in Table 4, all the equations have a postive exponent, which means that the circumference of individual tubules becomes smaller, but that this decrease is overcome by an increase in the number of tubules.

Circumference of individual Henle's thin segments (CIH)

The circumference of individual Henle's thin segments were constant and independent of $X$ in the animals or human case; man: $0.1008 \pm 0.0051 \mathrm{~mm}$; swine : $0.0400 \pm 0.0064 \mathrm{~mm}$; $\operatorname{dog}: 0.1040 \pm 0.0024 \mathrm{~mm}$; hamster : $0.065 \pm 0.0031$ $\mathrm{mm}$ at a level of $5 \%$.

Total circumference of Henle's thin segments (TCH)

A linear regression of the total circumferences of ascending or descending limbs of Henle's thin segments was clearly demonstrated on a semilogarithmic coordinate system in the animals or human case. As shown in Fig. 5, TCH was more than TCC towards the external border of the inner medulla in man and swine, but TCH was less than TCC throughout the entire length of the inner medulla in dog and hamster. These differences in the quantitative relation of TCC and TCH must be due to the structural differences in Henle's loops and the collecting tubules in the inner medulla described above. The equations obtained, expressed by functions of $X$, are shown in Table 5 .

TABLE 5. The equations of the total external circumference $L$ of Henle's thin segments expressed as a exponential function of $X$

\begin{tabular}{lll}
\hline Species & $L=A \mathrm{e}^{B X}(\mathrm{~mm})$ & $Y=B X+C(Y=\log L)$ \\
\hline Man & $L=18.68 \mathrm{e}^{0.55 X}$ & $Y=0.55 X+2.93$ \\
Swine & $L=18.01 \mathrm{e}^{0.56 X}$ & $Y=0.56 X+2.89$ \\
Dog & $L=27.70 \mathrm{e}^{0.75 X}$ & $Y=0.57 X+3.32$ \\
Hamster & $L=7.60 \mathrm{e}^{0.68 X}$ & $Y=0.68 X+2.03$ \\
\hline
\end{tabular}

$A, B$ and $C$ are constants.

\section{Discussion}

Since the countercurrent hypothesis was presented by Hargitay and Kuhn (1951) and Kuhn and Ramel (1959), it is generally accepted that the generation of a hypertonic medullary interstitium is the result of operation of a countercurrent multiplier system in Henle's loop. The countercurrent multiplier system is constituted on the assumption that the ascending thin segment of Henle's loop is 
the site of active reabsorption of sodium chloride and restricted hydraulic conductivity. The combination of these functional properties should lead to the removal of hypertonic material from the tubular lumen, rendering the tubular fluid hypotonic with respect to the adjacent interstitium. The final concentration of the urine is supposed to result from passive diffusion of water from the collecting tubules to the interstitium as the urine passes through regions of increasing osmotic pressure. However, the functions of the thin segment of Henle's loop have not been clarified. Since no histological differences between the ascending and descending parts of the thin segment have been demonstrated, it is difficult to conceive that sodium should be reabsorbed in the ascending thin segment and not in the descending thin segment. Furthermore, Marsh and Solomon (1965) obtained no experimental evidence of active salt reabsorbtion from either the thin ascending or thin descending segment in the hamster papilla. If this is true, the countercurrent multiplier theory does not hold in the inner medulla, and there must be some other mechanism for concentration of urine in this region.

Kokko and Rector (1972) and Stephenson (1972) independently proposed a passive model of urinary concentration that did not involve active transport of salt in the thin ascending segment of Henle's loop. In their models, Henle's loop is treated as an exchanger system of the diffusion type. According to Kokko and Rector, the thin descending segment of Henle's loop is relatively impermeant to solute but has high osmotic water permeability, while the thin ascending segment of Henle's loop is more permeant to $\mathrm{NaCl}$ than urea but is relatively impermeant to osmotic flow of water. This combination of permeability properties would permit addition of urea to the medullary interstitium from the collecting duct to promote the osmotic withdrawal of water from the thin descending segment of Henle's loop, thereby raising the intraluminal concentration of $\mathrm{NaCl}$ above that of the surrounding fluid, and then the diffusion of $\mathrm{NaCl}$ out of the thin ascending segment of Henle's loop down a concentration gradient to generate hypotonic intraluminal fluid. Thus, there is a difference of opinion about the function of the thin segment of Henle's loop in the inner medulla, and two models, 'active' and 'passive', for urinary concentration have been proposed. In both models, theoretical treatment of medullary physiology has been based entirely on a simplified model with a single Henle's loop and an unbranching collecting tubule, and the conclusions are directly correlated with experimental results. However, the renal medulla consists of a large number of Henle's loops of very different lengths and ramified collecting tubules, as shown in this study. Thus it is obvious that theoretical conclusions based on results on a single Henle's loop cannot be applied directly to complete medulla and to its overall function. The final sodium concentration of the interstitial tissue of the inner medulla is the result of the interaction of sodium and water transport from both Henle's loops and collecting tubules. The total quantity of sodium depends not only on the net flux of solutes or water of individual tubules but also on the numbers of Henle's loops and 
collecting tubules.

In this study, both the total circumference of collecting tubules (TCC) and that of Henle's thin segments (TCH) could be approximated by an exponential function of $X$ of the form of $L=A \mathrm{e}^{B X}$ in the animals or human case examined. The total circumferences of Henle's thin segments increases with increase in $X$. This increase is brought about by increase in the number of short loops, since the circumference of individual thin segments is constant. Results showed that TCH was more than TCC toward the external border of the inner medulla in man and swine. This finding indicates that in the pile of Henle's loop, short loops predominate over long ones. The rabbit kidney is structurally similar to those of man and swine. Sasaki, Takahashi and Suwa (1969) and Sasaki and Suwa (1969) calculated that this structural feature counteracts the effect of producing a gradient of sodium concentration towards the medullary apex on the basis of the countercurrent multiplier system. They regarded the Henle's thin segment as an exchanger, sodium depriving system. However, in contrast to man and swine, TCH was less than TCC along the entire length of the inner medulla in dog and hamster. Therefore, it is uncertain whether their hypothesis can be applied to these animals. Further mathematical studies are needed on this problem.

Ullrich et al. (1961) has reported that maximum urine osmolarity of man, dog and swine was in the range from $1,000 \mathrm{mOsm} / \mathrm{kg} \mathrm{H} \mathrm{H}_{2} \mathrm{O}$ to $2,000 \mathrm{mOsm} / \mathrm{kg} \mathrm{H}_{2} \mathrm{O}$ and the highest in dog, followed in order man and swine. In this study, maximal urine osmolarity of the hamsters was $2,303 \pm 187 \mathrm{mOsm} / \mathrm{kg} \mathrm{H}_{2} \mathrm{O}$ and the relative medullary thickness showed the highest in hamsters of all subjects examined (hamster: 2.27, $\operatorname{dog}: 1.48, \operatorname{man}: 1.38$, swine: 0.49). The facts indicate that the concentration ability of the urine is the highest in hamster, followed in order by dog, man and swine. On the other hand, the exponents of equations of TCH were nearly constant (hamster: 0.68 , dog: 0.75 , man: 0.55, swine : 0.56) but those of TCC increased with increase in ability to concentrate the urine (hamster : 0.92, dog: 0.57, man : 0.28, swine: 0.26) This indicates that there is no essential difference in structural principle of Henle's loops in the inner medulla in any of these species. An architectural difference in the kidneys of these species is found in the structure of the collecting tubules in the inner medulla. This fact suggests that the structure of the collecting tubules in the inner medulla is important in the regulatory mechanism of urinary concentration.

\section{References}

1) Hargitay, B. \& Kuhn, W. (1951) Das Multiplikationsprinzip als Grundlage der Harnkonzentrierung in der Niere. Z. Elektrochem., 55, 539-558.

2) Kokko, J.P. \& Rector, F.C., Jr. (1972) Countercurrent multiplication system without active transport in inner medulla. Kidney Int., 2, 214-223.

3) Kuhn, W. \& Ramel, A. (1959) Aktiver Salztransport als möglicher (und wahrscheinlicher) Einzeleffekt bei der Harnkonzentrierung in der Niere. Helv. chim. Acta., 42, $628-660$. 
4) Marsh, D.J. \& Solomon, S. (1965) Analysis of electrolyte movement in thin Henle's loops of hamster papilla. Amer. J. Physiol., 208, 1119-1128.

5) Sasaki, Y. \& Suwa, N. (1969) Functional model of inner medulla of rabbit kidney based on its structural principle. Tohoku J. exp. Med., 98, 33-63.

6) Sasaki, Y. Takahashi, T. \& Suwa, N. (1969) Quantitative structural analysis of the inner medulla of rabbit kidney. Tohoku J. exp. Med., 98, 21-32

7) Schmidt-Nielsen, B. \& O'Dell, R. (1961) Structure and concentrating mechanism in the mammalian kindey. Amer. J. Physiol., 200, 1119-1124.

8) Stephenson J.L. (1972) Concentration of urine in a central core model of the renal counterflow system. Kidney Int., 2, 85-94.

9) Ullrich, K.J., Kramer, K., \& Boylan, J.W. (1961) Present knowledge of the countercurrent system in the mammalian kidney. Prog. Cardiovasc. Dis., 3, 395-431. 